\title{
TagHunt: Uma plataforma Combinando a Internet das Coisas com Scavenger Hunt Games
}

\author{
Kiev Gama ${ }^{1}$, Rafael Wanderley ${ }^{12}$, Daniel Barbosa Maranhão ${ }^{1}$, Vinicius Garcia ${ }^{1}$ \\ ${ }^{1}$ Centro de Informática - Universidade Federal de Pernambuco (CIn/UFPE) \\ Recife-PE-Brasil \\ ${ }^{2}$ Centro de Estudos e Sistemas Avançados do Recife - C.E.S.A.R \\ Recife-PE-Brasil \\ $\{$ kiev, rrw, dbm2, vcg $@$ ecin.ufpe.br
}

\begin{abstract}
The "Internet of Things" (IoT) brings the notion of heterogeneous objects using ubiquitous technologies to interact among them and with the physical environment through technologies such as Bluetooth, ZigBee, GPRS, NFC, QR code, among others. Based on the possibility of linking ordinary objects from the physical world to the Internet, this paper proposes and details a platform called TagHunt, for creating and playing scavenger hunt games. This platform leverages on smartphones' capability to interact with ordinary objects using IoT-based technologies such as NFC and QR Code, stimulating the player to interact with physical environments looking for "clues" in the game.
\end{abstract}

Resumo. A "Internet das Coisas" (IoT) traz a noção de objetos heterogêneos utilizando tecnologias ubíquas para interagir entre si e com o ambiente físico por Bluetooth, ZigBee, GPRS, NFC, código QR, dentre outros. Com base na possibilidade de associar utensílios comuns do mundo físico para a Internet, este trabalho propõe e detalha uma plataforma chamada TagHunt, para criar e praticar jogos de caça ao tesouro. A plataforma aproveita a capacidade de smartphones para interagir com peças comuns, usando tecnologias baseadas em Internet das coisas, tais como NFC e QR, estimulando o jogador a interagir com objetos físicos à procura de "pistas" no jogo.

\section{Introdução}

A "Internet das Coisas" (Internet of Things - IoT) é um conceito inicialmente utilizado para se referir a uma rede em que objetos seriam identificados individualmente e instantaneamente por radiofrequência (RFID) (Ashton, 2009). A visão atual da comunidade acerca da IoT evoluiu para a perspectiva de um universo de dispositivos, objetos e coisas, interligados, através de tecnologias heterogêneas (Yan et al 2008), alcançando parcialmente a visão de computação ubíqua de Mark Weiser (Weiser, 1994). Além de RFID, diversas tecnologias sem fio servem de base para a Internet das coisas, tais como Bluetooth, ZigBee, GPRS, NFC, dentre outras (Zhou, 2012).

Cada vez mais, várias dessas tecnologias de IoT vêm sendo integradas a smartphones, motivando novas possibilidades na criação de aplicações dentro desse contexto. Elas viabilizam o conceito de object hyperlinking (Vazquez-Briseno et al, 2012), que consiste em criar representações virtuais de objetos físicos no mundo virtual. Exemplos comuns são 
etiquetas NFC (Near Field Communication) e QR Code fixadas em objetos reais, mas com URLs para sites, disponibilizando ações ou informações adicionais ligadas ao objeto real (ex: site de compra do objeto, manual online do objeto). O conceito vem sendo explorado, por exemplo, pelo Google no projeto Physical $\mathrm{Web}^{1}$, que propõe o uso de URLs para etiquetar objetos virtualmente.

Este artigo propõe um cenário lúdico para a IoT no contexto de object hyperlinking, permitindo uma espécie de realidade mista (Milgram e Kishino, 1994) em jogos digitais, envolvendo objetos do mundo físico em contexto urbano. Nesses jogos, os participantes vão usar smartphones para obter dados a partir de objetos do mundo real. Os dados guiarão os usuários nas diferentes etapas de um tipo de jogo, originalmente não digital, chamado scavenger hunt, que é uma espécie de caça ao tesouro. Em cada etapa, o usuário terá de recolher as pistas sobre o próximo passo. Na nossa proposta, as informações podem ser recolhidas a partir de diferentes tipos de objetos (coisas) interativos, alimentados por diferentes tecnologias (código NFC, QR, Bluetooth). Elas levam o jogador para outra fonte de informação (ex: um mapa, uma página da Web) sobre o próximo passo, ou pedem informações adicionais (ex: um questionário). A combinação de NFC com jogos de caça ao tesouro é algo tipicamente ad hoc. Propomos uma plataforma chamada TagHunt, que além de ser genérica, permite que os usuários não-especialistas criem os próprios jogos Scavenger Hunt, onde objetos podem ser concebidos de forma ilimitada, incluindo a tecnologia NFC, o código QR e Bluetooth Low Energy, como exemplos.

Um experimento com quatro jogos distintos de scavenger hunt foi realizado. Eles foram criados com a plataforma, totalizando 110 participantes. $O$ primeiro jogo se deu no Centro de Informática da UFPE, envolvendo uma população de 15 indivíduos. Os outros três jogos foram realizados dentro do festival de tecnologia "Campus Party Recife 2014", durante três dias, envolvendo 35, 22 e 38 indivíduos, respectivamente. Após executar o experimento com usuários convidados para jogar usando os próprios smartphones, foi possível coletar dados sobre o uso do jogo, bem como efetuar entrevistas com uma amostra significativa da população do experimento. Ao analisar os dados, pudemos avaliar o envolvimento dos usuários com os jogos. Após medir a atividade dos participantes nesses cenários, e analisar o engajamento e a percepção destes nos jogos, pudemos aplicar a abordagem em um contexto não-experimental, em um cenário urbano, precisamente no centro do Recife, considerando as descobertas do experimento.

Como principais contribuições deste trabalho, podem ser apontadas: (1) a noção de uma plataforma Web para a criação de jogos de scavenger hunt empregando object hyperlinking; (2) a avaliação do engajamento dos jogadores em jogos deste tipo. O restante deste artigo está organizado da seguinte forma: a Seção 2 fornece mais fundo sobre os principais conceitos utilizados neste trabalho e algumas motivações para a nossa experiência. Seção 2 introduz a proposta de plataforma e sua implementação. Seção 3 apresenta o experimento e discute resultados. Seção 4 descreve trabalhos relacionados; e finalmente, a Seção 5 apresenta as conclusões e trabalhos futuros sobre o assunto.

\section{A Plataforma TagHunt}

O ambiente proposto para a construção de jogos de scavenger hunt, mesclados com o conceito de object hyperlinking (Vazquez-Briseno et al, 2012), visa estimular a interação de pessoas com objetos do mundo físico em cenários tais como centros históricos, praças públicas ou mesmo ambientes fechados como shopping centers e centros de convenções. Através de um

\footnotetext{
${ }^{1}$ https://google.github.io/physical-web/
} 
smartphone, usuários executando uma aplicação móvel podem interagir com objetos equipados com tecnologias de IoT (elementos de interação) e recolher informação contextual a partir dele, para que seja útil para a próxima etapa do jogo. Por exemplo, depois de verificar uma pintura com uma etiqueta NFC oculta, o aplicativo móvel pode exibir informações (ou seja, uma "pista") para encontrar o próximo elemento de interação do jogo - ou simplesmente fornecer acesso a mais informações sobre o objeto (ex: conectar-se a um vídeo, a um questionário, exibir uma imagem).

Os principais conceitos desse ambiente são ilustrados na Figura 1.a, de acordo com diferentes elementos que são detalhados a seguir.

Modelagem de passos/pistas. Uma pessoa sem experiência em programação deve ser capaz de usar um navegador para acessar o editor visual hospedado em ambiente de computação em nuvem, para a modelagem de uma sequência de jogo e cada ação correspondente.

Mapeamento dos elementos físicos. Para cada etapa/ação do jogo é necessário fazer um mapeamento da tal fase e o objeto físico correspondente que dispara a ação (um elemento de interação NFC, QRCode, Bluetooth, etc). Atributos adicionais (ex: geoposicionamento) podem ser levados em conta no jogo, para inferir contexto.

Definição do jogo. Cada um deles é um conjunto de passos, ações ou pistas, onde é possível criar vários jogos na plataforma. Uma definição de jogo deve ser armazenado em um formato compreensível pela plataforma. Ele define os passos, ações ou pistas do jogo de passo e os respectivos elementos de interação.

Motor do jogo. O motor de jogo lista os jogos disponíveis, interpreta a definição de jogo, interage com aplicações móveis do usuário e atualiza o estado do jogo para cada usuário. $\mathrm{O}$ motor seguirá um modelo multi-tenant, executando no mesmo ambiente, sendo capaz de fornecer diferentes instâncias de motores de jogo para diferentes clientes.

Aplicação móvel. Com base nas interações do usuário (ex: digitalizar o ID de uma tag NFC), o aplicativo móvel comunica com o lado do servidor e fornece ao usuário um feedback (por exemplo, obter mais informações, mensagem de erro).

Interações e as coisas do mundo físico. As interações são o ato de se comunicar com objetos com tecnologia IoT a partir do mundo físico, podendo desencadear ações no jogo (ex: a reprodução de um vídeo, um quiz) ou simplesmente fornecer informações para a próxima etapa (ex: uma nova pista).

Estado do jogo. Cada usuário tem sua própria instância do jogo definido para armazenar o eprogresso do usuário no jogo. Cada ação ou passo disparado pode ser visto como o estado de uma máquina de estados. $\mathrm{O}$ andamento do jogo se comporta como uma máquina de estados finitos. 


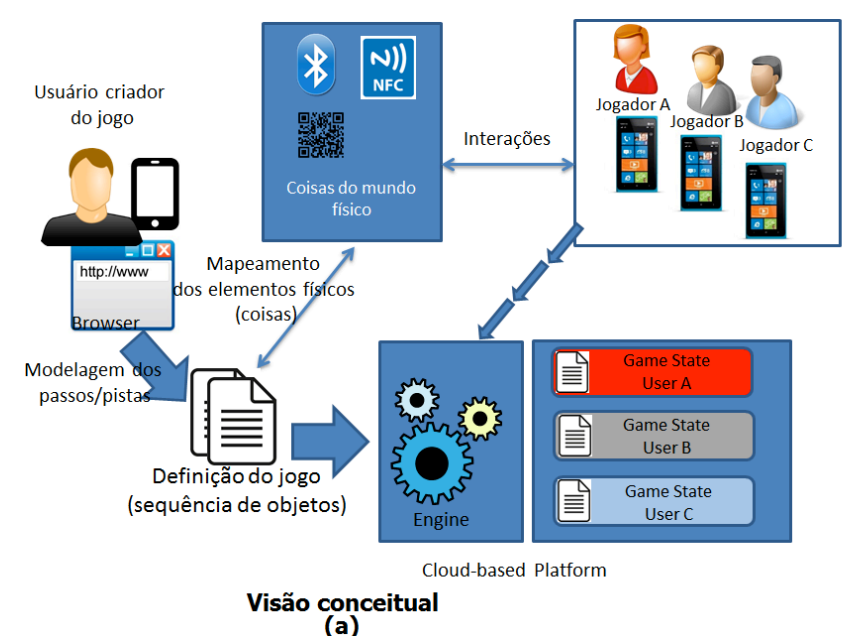

(a)

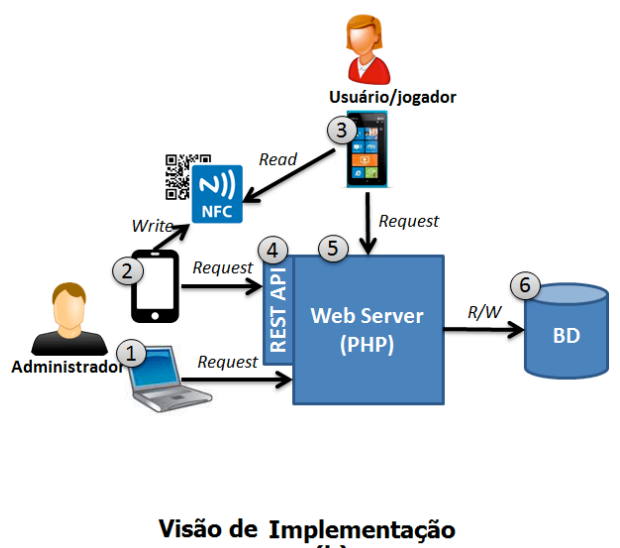

(b)

Figura 1. Visão conceitual e de implementação da plataforma

\subsection{Implementação}

Devido ao fato de a prova de conceito focar no engajamento de usuários, pensou-se nos seguintes requisitos: (1) a aplicação deve alcançar o máximo de dispositivos possível e (2) o jogo deve ser fácil de jogar, sem muitos passos adicionais, tais como registro ou login de usuário. Baseado nisso, decidiu-se uma abordagem focada em uma aplicação Web, onde nenhuma instalação App seria necessária, e não incluindo qualquer informação do usuário (jogadores anônimos identificados através de cookies), cumprindo, assim, ambos requisitos. Devido a essa escolha arquitetural de não usar um aplicativo, a implementação não incluía proximidade via Bluetooth, que necessitaria da API do smartphone e, consequentemente, necessitaria ser implementado como aplicação de plataforma específica (ex: Android). Cada pista de um jogo teria um ID único, representado por uma URL a ser codificada em uma etiqueta NFC ou QR code.

Uma visão geral da implementação está na Figura 1.b, cujos itens numerados (não necessariamente representando ordem de execução) são detalhados abaixo:

1. Cliente Web usado pelo administrador da plataforma de jogos.

2. App Android para gerenciamento de jogo, que lista ou jogos ou quests disponíveis (uma caça ao tesouro) e permite que o administrador do jogo possa escrever as tags NFC com os respectivos URLs únicas de cada pista.

3. Aparelho lê etiqueta NFC ou QR que contém a URL de uma pista e exibe a respectiva página contendo a informação (a Figura 2 ilustra a coleta de uma pista final).

4. API REST que permite o App Android de gerenciamento do jogo consultar o servidor sobre as missões disponíveis e as respectivas etapas de jogo (por exemplo, pistas).

5. Servidor Web PHP que contém as regras de negócios para a criação de busca e implementação motor de jogo.

6. Banco de dados onde as informações de busca e progresso do usuário são armazenados.

Para ilustrar o fluxo de criação de jogo, pode-se pensar em um usuário que é responsável por acessar o aplicativo Web administrativo. Esta é a interface do usuário, para a criação de um jogo de caça ao tesouro (ou quest). Para cada missão, o usuário pode adicionar novas pistas (ou passos). Durante o jogo, cada pista corresponde a uma URL única. Após criar todas as pistas necessárias, o usuário administrador do jogo pode usar uma aplicação móvel específica (Android), que irá interagir com o servidor Web e listar as quests disponíveis. 
Depois de escolher uma busca, a aplicação exibe a respectiva lista de pistas disponíveis para aquela pesquisa. O App dá a opção de escrever uma URL única da pista em uma tag NFC, usando a API NFC do Android.

O aplicativo Web foi hospedado em uma instância Jelastic Cloud ${ }^{2}$ do provedor Locaweb. Quando acessado durante o jogo, a URL com a pista era exibida pelo browser nativo do smartphone, direcionando o usuário para aplicativo Web conforme exemplo da Figura 2. Opcionalmente, o usuário também pode acessar o sistema para gerar o QR Code contendo a URL de uma pista. Usuários que começam jogando são anônimos, sem qualquer necessidade de login ou de se identificar nos sistemas. Um usuário é identificado exclusivamente graças a cookies do navegador, que permitem que a plataforma baseada na Web monitore o progresso em uma determinada caça (scavenger hunt), armazenando informações sobre quais pistas o usuário tenha coletado.

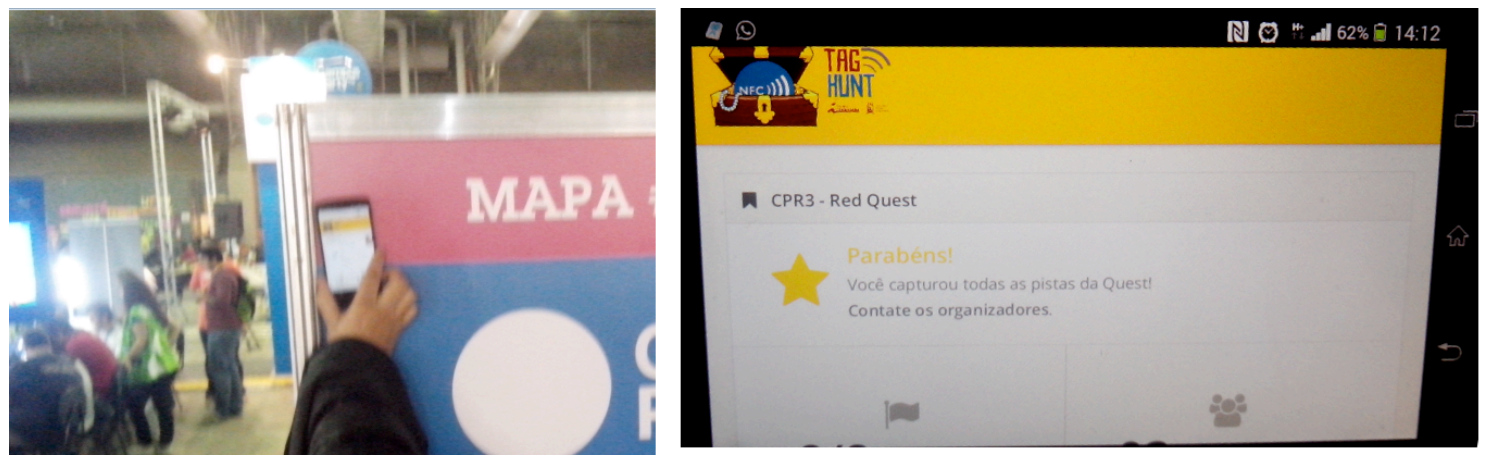

Figura 2. Leitura de etiqueta NFC escondida e screenshot da aplicação Web

\section{Experimentos}

Para validar a plataforma, foram criados quatro jogos diferentes de caça ao tesouro, com TagHunt. Em cada um deles foram coletadas informações de maneira implícita, utilizando o sistema na Web para coletar progresso do usuário, e, explicitamente através de questionário em papel, após conclusão de participação.

Os objetivos do experimento foram dois: (1) validar a plataforma, usando-o para criar jogos reais de caça de pistas e (2) convidando usuários para testar jogos criados com a nossa plataforma, para avaliar a experiência do usuário. Embora a implementação atual do jogo trabalhasse tanto com NFC e QR Code, os experimentos focaram exclusivamente no uso de etiquetas NFC, como a tecnologia da Internet das Coisas, usando o conceito de object hyperlinking. Tags NFC foram colocadas escondidas atrás de objetos finos (ex: cartazes, banners, letreiros) ou coladas sobre os objetos visíveis (ex: contêineres plásticos de lixo, paredes), como ilustrado no lado esquerdo da Figura 2.

Para modelar as questões a serem utilizadas nos questionários, alguns valores fundamentais foram tomados em consideração, seguindo diretrizes descritas por Kaasinen (2005): Facilidade de adoção, Valor percebido, Confiança e Facilidade de Uso Percebida.

Nove (9) perguntas, conforme descrito na Tabela 1, foram respondidas de acordo com uma escala de Likert típico de 5 itens (Concordo totalmente, concordo parcialmente, indiferente, discordo parcialmente, discordo totalmente). Q1 a Q3 estavam focadas na Facilidade de Adoção, Q4 Q5 com o valor percebido, Q6 e Q7 foram relacionados com confiança, e Q8 e Q9 estavam focados na percepção de facilidade de uso.

\footnotetext{
${ }^{2} \mathrm{http}: / /$ jelastic.com
} 
Tabela 1. Questionário aplicado após participação no jogo

Q1. As atividades propostas pelo jogo eram claras e os objetivos foram suficientes para a realização de tais atividades.

Q2. Foi fácil encontrar as tags NFC com base nas informações jogo.

Q3. As informações disponíveis no smartphone eram legíveis e bem posicionadas na tela.

Q4. Durante o jogo me senti motivado para executar os objetivos propostos.

Q5. Gostaria de usar este jogo em um espaço público (por exemplo, em um parque, no meu bairro, na minha cidade)

Q6. O conjunto inicial de instruções (configuração do smartphone, como ler uma tag NFC, entre outros) foram suficientes para a realização das atividades do jogo.

Q7. As informações fornecidas no jogo foram suficientes para executar a próxima etapa das atividades.

Q8. Eu me senti confortável em usar a tecnologia NFC em jogos ou aplicações.

Q9. Gostaria de usar novamente um jogo ou aplicativo que utiliza a tecnologia NFC.

\subsection{População}

A população do experimento consistiu em usuários familiarizados com a tecnologia. $\mathrm{O}$ primeiro jogo envolveu 15 alunos de cursos de graduação e pós-graduação do Centro de Informática da UFPE. Os demais foram realizados na Campus Party Recife, envolvendo um público mais diversificado, composto por entusiastas de tecnologia. A tabela 2 resume os dados da população do experimento.

Tabela 2. População participante do experimento

\begin{tabular}{|c|c|c|c|c|}
\hline Experimento & $\begin{array}{l}\text { Total de } \\
\text { participantes }\end{array}$ & Respondentes do questionário & Média de idade & Desvio padrão \\
\hline$\# 1$ & 15 & 15 & 24.6 & 5.5 \\
\hline$\# 2$ & 35 & 22 & 21.9 & 4.0 \\
\hline \#3 & 22 & 8 & 21.1 & 2.4 \\
\hline \#4 & 38 & 11 & 24.1 & 5.5 \\
\hline
\end{tabular}

\subsection{Dados}

No experimento 1 (CIn-UFPE) verificou-se que apenas 10 usuários $(66,7 \%)$ completaram a missão proposta (inicialmente grupo de 15 usuários). O experimento 2 iniciou-se com 35 usuários e $19(54,3 \%)$ concluíram. O experimento 3 teve 22 participantes e 9 deles $(40,9 \%)$ finalizaram a missão proposta. No experimento 4 foram inicialmente registados 38 usuários e $9(23,7 \%)$ acabaram coletando todos os tags. Estes números são ilustrados na figura $3 \mathrm{a}$, onde pode ser notado que os experimentos 3 e 4 tiveram oito etiquetas (i.e, pistas), enquanto experimentos 1 e 2 envolveram a coleta de 6 etiquetas. Em relação aos modelos de smartphones utilizados, Android e Windows Phone foram observados como os modelos mais populares entre os 110 jogadores, com um total de 90 (81,8\%) e 19 (17,2\%) telefones, respectivamente. A exceção foi um Blackberry que participou do experimento 1 , ou seja, menos de $1 \%$ em todos os experimentos.

\subsection{Discussão}

A exigência de o aplicativo funcionar no máximo de plataformas possíveis era de fato uma escolha importante que levou a uma abordagem baseada na Web. Essa decisão permitiu que telefones equipados de NFC - Android e Windows Phone - participassem dos jogos, sem qualquer necessidade de instalação de aplicativos específicos, viabilizando um verdadeiro cenário touch and play.

O gráfico de linhas apresentado na Figura 3a ajuda a visualizar as tendências na taxa de abandono no jogo. $\mathrm{O}$ eixo $\mathrm{Y}$ mostra o número de jogadores que fizeram a coleta e o eixo $\mathrm{X}$ 
enumera cada pista do jogo. Por exemplo, a pista 1 sempre vai ser contabilizada para todos os participantes em uma caçada, uma vez que consideramos que o usuário já coletou pelo menos uma etiqueta. Já a pista 2, só será contabilizada por aqueles que a encontrarem. No experimento 4, a URL para a primeira pista foi informada via redes sociais, alcançando mais de 100 pessoas acessando a URL. Ao usar o cabeçalho HTTP_REFERER a partir da requisição HTTP, foi possível identificar os falsos positivos, tais como sistemas operacionais sem suporte a NFC (ex: Apple iPhone). Após a filtragem, apenas 38 usuários foram considerados como participantes desse experimento.

O bounce-rate, que representa quando os usuários desistiam, é visível na Fig. 3a. Ela mostra a estabilização entre as pistas de números 4 e 6 , quando, aparentemente, os usuários seguem engajados . Nas experiências 3 e 4, o número de usuários começa a cair novamente nas pistas 7 e 8 . O gráfico sugere que jogos de caça ao tesouro tenham entre 4 e 6 pistas, em sua totalidade, para manter o engajamento dos usuários. Contudo, mais investigações seriam necessárias, uma vez que há outras variáveis, como a dificuldade de encontrar o objeto escondido - o que representa uma ameaça à validade do experimento.

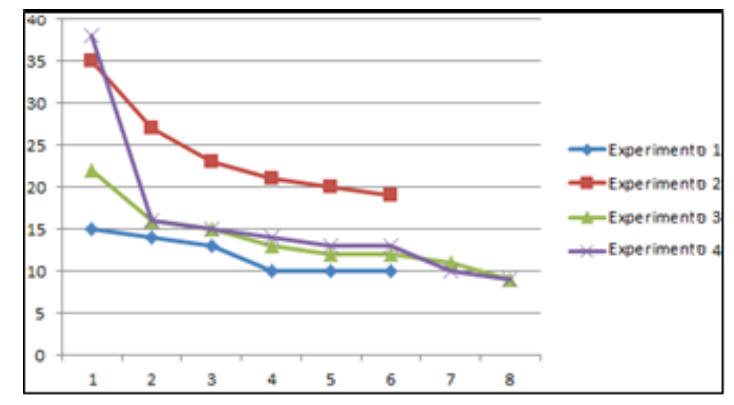

Etiquetas coletadas pelos usuários

(a)

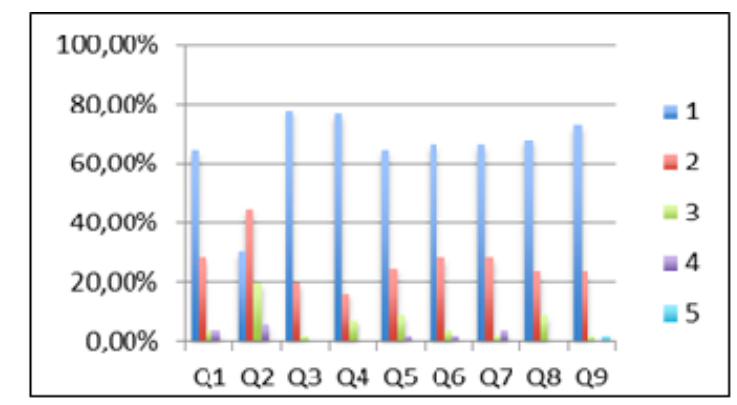

Respostas dos questionários

(b)

Figura 3. Dados dos experimentos

A fig. $3 \mathrm{~b}$ ajuda a compreender o fato. Todas as questões trazem mais de $60 \%$ das respostas como Concordo Totalmente, com exceção de Q2, que não segue o padrão. A questão diz respeito à "Facilidade de adoção" ser especificamente sobre a dificuldade de encontrar as tags NFC baseados em informações do jogo. Ainda sobre essa questão, cerca de $30 \%$ disseram Concordar Totalmente e mais de $40 \%$ concordaram parcialmente, enquanto $30 \%$ se mantiveram indiferentes. Normalmente, nas outras perguntas, respostas neutras ficaram abaixo de $10 \%$. Também analisamos cada experimento individualmente e um padrão muito semelhante pode ser identificado sobre a Q2, diferindo de outras respostas.

O cenário motivador da proposta deste projeto seria em um ambiente urbano, para permitir que as pessoas explorem e interajam com a cidade. Configuramos um jogo de scavenger hunt em uma área turística do centro do Recife, com o apoio da prefeitura da cidade, no contexto de um trekking urbano, conforme reportado pela mídia local ${ }^{3}$. Os principais pontos adaptados neste scavenger hunt foram utilizar o limite de 6 pistas, baseado nas conclusões dos experimentos, e a inclusão de QR code, aumentando o escopo de dispositivos possíveis, com a participação de usuários de dispositivos sem NFC.

\footnotetext{
${ }^{3}$ http://www.leiaja.com/tecnologia/2014/09/28/cacada-tecnologica-no-recife-antigo-de-coracao/
} 


\section{Trabalhos relacionados}

\subsection{Object hyperlinking com NFC e RFID}

Antes da padronização da tecnologia NFC, jogos experimentais com telefones dotados de RFID (Rashid et al, 2006) já vinham empregando o conceito de realidade mista (Milgram e Kishino, 1994). Este consiste nos mundos reais e virtuais se fundindo para produzir um novo ambiente, onde os objetos físicos e digitais podem coexistir e interagir. $\mathrm{O}$ uso recente de aparelhos NFC em cenários interativos tem sido basicamente focado em locais de exposição (ex: museus, feiras), como apresentado por Rudametkin et al (2010) e Pesonen e Horster (2012). A ideia de utilizar NFC para estimular a interação com objetos de meio ambiente do usuário tem sido explorado na investigação. Isto é especialmente verdadeiro em ambientes urbanos. Por exemplo, cartazes inteligentes (smart posters) com NFC foram usados como fonte de informação em locais turísticos (Borrego-Jaraba et al, 2011). Em outra abordagem, a tecnologia foi usada para construir uma ferramenta onde os investigadores tentaram combinar projeção de imagem com tags NFC em demonstrações públicas interativas (Broll et al, 2011).

Apenas em alguns desses cenários introduz-se a noção de jogos. Por exemplo, no projeto "Touch \& Collect" (Riekki, 2007), os visitantes podem escolher a URL tocando as tags NFC para exibir itens com seus celulares NFC. Os visitantes podem jogar depois do conteúdo, quer em seus telefones móveis, em computadores domésticos ou ainda em telas espalhadas pelo museu. Outro projeto, chamado PLUG (Gentes et al, 2011), experimentou aparelhos habilitados para NFC para um jogo onde os usuários podem escolher cartões armazenados em tags NFC espalhadas pelo museu e, em seguida, ser capaz de trocar cartões usando o modo de NFC P2P. No entanto, como pudemos verificar, ambientes de jogo no contexto da RFID e NFC consistem tipicamente de cenários pré-definidos, sem qualquer flexibilidade para alterar ou criar novas configurações - o que é proposto com a plataforma TagHunt aqui apresentada.

Em cenários de ensino, no qual usuários de aplicações são tipicamente estudantes que interagem com seu ambiente, NFC tem sido combinado com gamification em situações de aprendizagem. A abordagem proposta por Sánchez et al. (2011) ajuda a aprender em dois cenários (idiomas e leitura), onde as crianças usam telefones equipados com NFC para interagir com os objetos marcados (Garrido et al, 2011). O trabalho de Kranz et al. (2013) tenta avaliar a popularidade de NFC com um jogo para smartphones Android. Usuários baixam o App que permite digitalizar etiquetas NFC aleatórias encontradas no seu dia a dia. Os autores empregaram gamification para estimular seus usuários a coletar tags e acumular pontos para cada tag "recolhida", com pontos de bônus para os que enviarem fotos das marcas, ou quando foram digitalizadas tipos raros de tags.

\subsection{NFC e Scavenger Hunt Games}

Jogos de coleta usando NFC podem ser encontrados em iniciativas da indústria, apesar de não fornecerem aos usuários a capacidade de criarem suas próprias missões como no caso do TagHunt. Um exemplo de tal tipo de iniciativa em torno do NFC é o Google I/O Hunt ${ }^{4}$, que foi disputado pelos participantes do evento Google I/O, em 2013, e mais tarde lançado como um projeto open source. Ao usar um aplicativo Android, os jogadores efetuavam leitura de etiquetas NFC que forneceram pistas para as outras tags. O objetivo do jogo era encontrar um cão oculto chamado Alex. O jogador interage com o jogo ao ler uma tag NFC ou responder questões de trivia.

\footnotetext{
${ }^{4}$ http://nfchunt.appspot.com/
} 
Munzee $^{5}$ é um jogo de caça ao tesouro, onde é possível procurar objetos no mundo real. Os itens são praticamente coletados por meio de smartphones. Usando uma abordagem gamification, o usuário ganha distintivos para os itens (chamados munzees) que recolhem, aumentando seu nível. Um munzee pode ter diferentes formas, mas se estiver equipado com uma etiqueta NFC ou um código de QR. Como parte do modelo de negócios da empresa por trás Munzee, eles permitem que os usuários implantem seus próprios munzees com a venda de tags NFC ou através de impressão (código QR).

A abordagem que encontramos como uma plataforma para criação de jogo em um cenário de computação pervasiva é bastante baseada em frameworks e APIs, em vez de permitir que as pessoas - que não são necessariamente os programadores - construam seus próprios jogos, que é a nossa proposta. Além disso, essa plataforma não lida diretamente com NFC. Na verdade, Placechallenge ${ }^{6}$ fornece uma plataforma para jogos baseados em localização que suportam jogos colaborativos e competição entre os jogadores. Dois exemplos de aplicações construídas utilizando esta plataforma são GetChi ${ }^{7}$ e Runaway ${ }^{8}$.

\section{Conclusões e Trabalhos Futuros}

Muitas tecnologias relacionadas com a Internet das coisas estão presentes em smartphones, incluindo NFC. Estamos motivados pelo uso de object hyperlinking para impulsionar o uso de NFC, e, alternativamente, QR Code, mesclando esta abordagem com jogos de scavenger hunt. Este trabalho propõe um novo cenário para a Internet das coisas, envolvendo objetos do mundo físico em um jogo digital. Nesses jogos, os participantes usam smartphones para obter dados a partir de objetos do mundo real. Os dados guiarão os usuários, com diferentes etapas de um jogo de caça ao tesouro. Nós trouxemos a noção de uma plataforma para a criação de jogos de caça ao tesouro que empregam object hyperlinking e é capaz de avaliar o envolvimento dos usuários nesses cenários.

Após a realização de quatro experimentos em dois locais diferentes, pudemos verificar que uma abordagem baseada na Web torna mais fácil para os usuários participarem dos jogos tipo caça ao tesouro usando a plataforma TagHunt, permitindo uma verdadeira abordagem touch and play. Depois de analisar os dados do jogo, verificamos que a taxa de usuários, se estabilizou entre 4 e 6 marcas em uma caçada. Este número pode estar relacionado à dificuldade de encontrar as palavras escondidas, necessitando, portanto, mais investigação. Outro fator importante observado é que, apesar de NFC ser relativamente simples de usar, verificou-se que a ausência de instruções para usuários explicando como jogar um jogo de caça ao tesouro usando NFC pode reduzir significativamente o número de jogadores que se envolvem na competição. Pretendemos construir aplicações nativas em smartphones (Android e iOS) para utilizar recursos como geolocalização e Bluetooth como elementos interativos.

\section{Agradecimentos}

Este trabalho contou com o apoio do INES (Instituto Nacional de Engenharia de Software), Emprel, Prefeitura do Recife e Campus Party Recife.

\footnotetext{
${ }^{5} \mathrm{http}: / /$ www.munzee.com/

${ }^{6} \mathrm{http}: / / \mathrm{www}$. placechallenge.com/

${ }^{7}$ http://getchiapp.com/

${ }^{8} \mathrm{http}: / /$ runawayapp.com
} 


\section{Referências}

Ashton, K. That "Internet of Things" thing, RFID Journal (2009)

Borrego-Jaraba, F., Ruiz, I. L., \& Gómez-Nieto, M. Á. (2011). A NFC-based pervasive solution for city touristic surfing. Personal and ubiquitous Computing, 15(7), 731-742.

Broll, G., Graebsch, R., Scherr, M., Boring, S., Holleis, P., \& Wagner, M. (2011). Touch to Play--Exploring Touch-Based Mobile Interaction with Public Displays. In Near Field Communication (NFC), 2011 3rd Intl Workshop on (pp. 15-20). IEEE.

Garrido, P. C., Miraz, G. M., Ruiz, I. L., \& Gomez-Nieto, M. A. (2011). Use of NFC-based pervasive games for encouraging learning and student motivation. In Near Field Communication (NFC), 2011 3rd Intl. Workshop on (pp. 32-37). IEEE.

Gentes A., Jutant C., Guyot A., Simatic M., RFID Technology: Fostering Human Interactions, IADIS International Conference Game and Entertainment Technologies 2009. Carvoeiro. Portugal.

Kaasinen, E. (2005) User acceptance of mobile services value, ease of use, trust and ease of adoption. Espoo. VTT Publications 566. 151 p. + app. 64 p.

Kranz, M., Murmann, L., \& Michahelles, F. (2013). Research in the Large: Challenges for Large-Scale Mobile Application research-A Case Study about NFC Adoption using Gamification via an App Store. International Journal of Mobile Human Computer Interaction (IJMHCI), 5(1), 45-61.

Milgram, P., \& Kishino, F. (1994). A taxonomy of mixed reality visual displays. IEICE TRANSACTIONS on Information and Systems, 77(12), 1321-1329.

Pesonen, J and Horster, E., "Near field communication technology in tourism," Tourism Management Perspectives, vol. 4, pp. 11-18, Oct. 2012.

Rashid, O., Bamford, W., Coulton, P., Edwards, R., \& Scheible, J. (2006). PAC-LAN: mixedreality gaming with RFID-enabled mobile phones. Computers in Entertainment (CIE), 4(4), 4.

Riekki, J (2007). RFID and smart spaces, Intl. Journal of Internet Protocol Technology, Vol 2, No 3-4, 2007

Rudametkin, W, et al. "Towards a dynamic and extensible middleware for enhancing exhibits." (2010) Consumer Communications and Networking Conference (CCNC), 2010 7th IEEE. IEEE, 2010.

Sánchez, I., Cortés, M., Riekki, J., \& Oja, M. (2011). NFC-based interactive learning environments for children. In Proceedings of the 10th international conference on interaction design and children (pp. 205-208). ACM.

Vazquez-Briseno, M., Hirata, F. I., de Dios Sanchez-Lopez, J., Jimenez-Garcia, E., NavarroCota, C., \& Nieto-Hipolito, J. I. (2012). Using RFID/NFC and QR-Code in Mobile Phones to Link the Physical and the Digital World. Interactive Multimedia. Dr. Ioannis Deliyannis (Ed.) InTech, 219-242.

Weiser, M.. "Ubiquitous computing." (1994). ACM Conference on Computer Science. 1994.

Yan, L., Zhang, Y., Yang, L.T, Ning, H. (2008). The Internet of Things: from RFID to the Next-Generation Pervasive Networked Systems. Auerbach Publications, 2008.

Zhou, H. (2012). The Internet of Things in the Cloud: A Middleware Perspective. CRC Press, 2012 